Revisión

\title{
La Dehidroepiandrosterona (DHEA), revisión de su eficacia en el manejo de la disminución de la libido y de otros sintomas del envejecimiento
}

\author{
Jesús M. Mendivil Dacal, Valeria M. Borges \\ Servicio de Urología. Clínica de la Asunción. Tolosa, Guipúzcoa, España
}

\begin{abstract}
Resumen
Introducción: La Dehidroepiandrosterona, ampliamente utilizada para el control de los síntomas asociados al envejecimiento, ha sido objeto de numerosos ensayos clínicos con resultados discordantes en cuanto a su eficacia.

Objetivos: La presente revisión pretende analizar la información existente mediante una búsqueda y revisión sistemática de artículos centrados en la utilización de la DHEA/S para el control de los síntomas del envejecimiento.

Material y métodos: Revisión sistemática de la literatura y aplicación de las técnicas de metaánalisis para valorar la eficacia de la DHEA/S, para ello se normaliza el proceso de forma prospectiva, se revisaron 152 artículos y se utilizaron únicamente 25 para agregar sus resultados.

Resultados: Como único dato destacable, parece afectar al metabolismo de los hidratos de carbono aumentando la eficacia de la insulina.

Conclusión: Los estudios no sustentan la utilidad de la DHEA para la reducción de los síntomas asociados al envejecimiento.
\end{abstract}

Palabras clave: Dehidroepiandrosterona. DHEA. DHEAS. Libido. Envejecimiento.

\section{Dehydroepiandrosterone (DHEA), review of its efficiency in the managing of the libido decrease and other symtoms of the aging}

\begin{abstract}
Introduction: The DHEA widely used for the control of the symptoms associated with the aging, has been an object of numerous clinical essays with discordant results as for its efficiency.

Objectives: The present review tries to analyze the existing information by means of a search and systematic review of articles centred on the utilization of the DHEA/S for the control of the aging symptoms.

Material and methods: Systematic review of the literature and application of the skills of metaanalisis to value the efficiency of the DHEA/S, for it settles down the process of prospective form, 152 articles were checked and 25 were in use for adding only their results.

Results: Since the only (unique) prominent information seems to concern the metabolism of the carbohydrates increasing the efficiency of the insulin.

Conclusions: The studies do not sustain the usefulness of the DHEA for the reduction of the symptoms associated with the aging.
\end{abstract}

Keywords: Dehydroepiandrosterone. DHEA. DHEAS. Libido. Aging.

$\mathrm{L}^{2}$ a utilización de la DHEA como suplemento dietético es muy popular entre los colectivos que buscan desarrollo muscular y mejora en las funciones que se deterioran con el envejecimiento. Es muy conocida en el campo de la herboristería y medicina "natural" en donde sus indicaciones y dosificación se realizan de forma empírica.

La fuente natural de la DHEA es una planta de la familia de la Dioscóreas, la "Dioscorea villosa", que en sus raíces y rizomas contiene diosgenina, precursor natural de un gran numero de esteroides.
No obstante, la ingestión del precursor, no se sabe que en el organismo produzca DHEA. Son necesarios varios pasos industriales para obtener la hormona a partir de la diosgenina a pesar de lo cual, los extractos de la planta, se utilizan con los mismos fines que el producto purificado.

Se publicita para aumentar el vigor sexual, mejorar la percepción de estado de salud, aumento de la masa muscular, regulación de los niveles de lípidos y control de la diabetes tipo II. 


\section{Justificación del proyecto}

La Dehidroepiandrosterona (DHEA) y su derivada sulfatada (DHEAS) son los esteroides más abundantes en el plasma de ambos sexos. Son neuroesteroides y pueden ser sintetizados tanto en el cerebro como en la zona reticular del córtex adrenal.

El feto sintetiza una elevada cantidad de DHEA en la glándula adrenal pero, esta producción, decrece bruscamente tras el nacimiento para volver a aumentar de forma rápida a los 6-8 años y alcanzar el pico más elevado a los 25-30 años. Después se produce una caída de los niveles hasta llegar a la década de los 80 con un $10-20 \%$ de los valores pico $^{2}$.

No se conoce la acción fisiológica de la hormona DHEA en su totalidad. La DHEA es un substrato para la síntesis de la androsterona y de la testosterona; su aparición se acompaña de vello púbico y axilar lo que sugiere que, una de las acciones de la DHEA, es la de ser un andrógeno adrenal. Pero también hay pruebas de que actúa en el SNC. Es un antagonista del receptor del ácido gamma amino butírico (GABA), inhibidor de la neurotrasmision ${ }^{3}$, y un agonista del efecto neurotrasmisor del glutamato ${ }^{8}$. Se considera que actúa como un agente neuroprotector $^{5}$ que, junto a una acción estimulante sobre el sistema inmunológico ${ }^{9}$, se opone a la acción de los glucocorticoides que inducen la involución tímica, o a la acción neurotóxica del exceso de corticosteroides sobre todo en el hipocampo ${ }^{4,5,7}$.Por esto, en los adultos jóvenes, los efectos adversos del cortisol (por ej: en el stress o en un traumatismo) pueden ser compensados por los altos niveles de DHEA(S), cosa que no ocurre en los adultos viejos en quienes sus bajos niveles les dejan muy vulnerables al daño neuronal del cortisol ${ }^{10}$.

Hasta hoy no han podido ser identificados receptores que medien específicamente la acción de la DHEA y también queda por determinar si su acción es en la superficie de la célula o intracelular. Su acción parece estar ocasionada por sus derivados androgénicos y estrógenos en sus receptores específicos.

Existen algunas pruebas epidemiológicas de que niveles elevados de DHEA(S) en suero de hombres pueden protegerles de las enfermedades cardiacas e incrementar su longevidad ${ }^{11}$. Hay algunas evidencias clínicas y experimentales de que la administración de DHEA estimula la actividad natural de las células natural Killer9 mostrando así su efectividad en el tratamiento de las enfermedades inmunológicas $^{12,13}$. Algunos estudios muestran disminución de niveles en pacientes con demencia ${ }^{14-17}$, pero otros no encuentran esta diferencia ${ }^{18,19}$.

Se ha sugerido utilizar los niveles de DHEA/S en plasma como biomarcador del envejecimiento saludable.

Muller $\mathrm{M}^{35}$ confirma que los niveles de andrógenos y DHEA están correlacionados en varones y se relacionan con la actividad física, sexual y cognitiva.

Los posibles efectos pretendidos de la DHEA van desde su acción vasodilatadora, antienvejecimiento, antiinflamatoria, antiarteriosclerótica y se vende al publico como complemento energético. Aun existe discusión sobre los efectos y los receptores de la hormona pero se acepta que puede actuar en cinco niveles distintos:

1. La DHEA y DHEAS actúan sobre receptores GABA-A/Benzodiacepinico, neutralizando los efectos GABA.

2. En los receptores N-methyl-aspartato potencian las acciones del glutamato.

3. En el receptor nuclear CAR, en el que los metabolitos de la DHEA pueden actuar como facilitadores.

4. En los receptores de superficie de las células endoteliales, donde solo actúa la DHEA y no la DHEAS.

5. En los receptores Sigma-1 de los neuroestoroides, donde ejerce un efecto antidepresivo.

Con este perfil farmacológico, los usos clínicos que ha tenido la DHEA han sido en mujeres con deficiencia suprarrenal donde se produce un déficit androgenico, ya que la corteza suprarrenal es la única fuente de andrógenos. En la mujer no se conocen los efectos que el déficit de andrógenos pueda ocasionar.

El tratamiento con DHEA ha sido realizado para mejorar la libido, la depresión o la ansiedad, la mejora de la actividad física (a través del incremento de la masa muscular), también en el envejecimiento (tanto por la supuesta mejora en el nivel cognitivo como por la percepción de bienestar); en el control de la grasa abdominal a través del bloqueo del 11B-HSD1, reduciendo el paso de cortisona a cortisol y produciendo un incremento de la sensibilidad a la insulina.

La DHEA no está exenta de efectos secundarios, aparte de la androgenizacion, vello, acne, etc, produce elevaciones de HDL. Teóricamente resultaría 
de riesgo en pacientes con tumor de próstata, y se han descrito casos de manía o palpitaciones.

La dosis de tratamiento para alcanzar niveles similares al rango de normalidad encontrado en varones de 15 a 39 años es de $50 \mathrm{mg}$ al día por vía oral, suficiente para alcanzar los niveles deseados ${ }^{20}$ aunque es necesario que el tratamiento sea prolongado, meses, para lograr algunos de sus efectos.

El uso de la DHEA, su dosis, pautas de administración y control de efectos secundarios en cada uno de sus objetivos terapéuticos no están claros y aun se duda de su eficacia en algunas de sus indicaciones terapéuticas.

Los efectos secundarios no están bien estudiados pero se recomienda evitar el producto en aquellos procesos en los que un efecto androgénico pueda suponer un riesgo, como es el caso del cáncer de próstata.

Las incertidumbres en cuanto a su eficacia abren un espacio para revisar los estudios clínicos existentes sobre el tratamiento en humanos con DHEA en vista de los posibles beneficios teóricos sobre el envejecimiento. Se han publicado guías para su uso en función de la información existente en la actualidad ${ }^{28}$.

Específicamente los objetivos del estudio se centran en valorar su eficacia en:

- Modificación de la Masa Ósea

- Modificación de la Masa Muscular

- Cambios en los perfiles lipidicos

- Percepción de bienestar de forma global y en:

1. Memoria

2. Actividad sexual

3. Humor

Para alcanzar los objetivos señalados se plantea realizar una revisión sistemática de la literatura con la posterior realización de metaánalisis en los grupos de publicaciones que lo permitan.

\section{MATERIAL Y MÉTODOS}

Para la revisión sistemática de la literatura y aplicación de las técnicas de metaánalisis que permita la estimación de la eficacia de la DHEA/S se normaliza el proceso de forma prospectiva y se plantea el siguiente esquema de trabajo:

Búsqueda de revisiones sistemáticas: Utilización de las revisiones Chocrane y Uptodate en el uso de la DHEA/S y definir sobre qué medidas de resultado se debería centrar el estudio.

\section{Búsqueda de información sobre ensayos clínicos}

a. Dos niveles de búsqueda

i. En relación a la DHEA como hormona, centrándose en aspectos básicos de conocimiento de la misma que se utilizarán en la introducción del proyecto.

ii. En relación a la eficiencia de la DHEA como tratamiento antienvejecimiento, que formará parte de la información que se utilizará en el meta-análisis.

Las búsquedas se realizan utilizando una metodología de búsqueda y revisión estándar ${ }^{21,22}$. Se ha utilizado la base bibliografía Medline con los buscadores "PubMed". y "Ovid".

Las búsquedas se han realizado utilizando las siguientes palabras clave: "DHEA", "DHEAS", "Dehidroepiandrosterona” y con el 2006 como fecha limite. Con los siguientes parámetros de búsqueda

Field: Title/Abstract,

Límites: Middle Aged: 45-64 years, only items with abstracts, Clinical Trial, Humans.

Medidas de resultado: Se centran el estudio en aquellas publicaciones cuya medida de resultado tiene que ver con mejora en la percepción del estado físico, aspectos cognitivos, cambios en los perfiles lipidicos, masa muscular, masa ósea o actividad sexual.

\section{Creación de una metodologia de selección de los artículos}

Se diseña de forma prospectiva los criterios a seguir para la selección de los artículos en función de los objetivos planteados y su calidad.

Mecánica utilizada para la selección de los estudios:

Todos los estudios encontrados fueron revisados inicialmente por un único revisor utilizando la información contenida en el resumen y se desecharon todos aquellos que resultaron irrelevantes para los objetivos de la revisión o que no correspondían a medidas de resultado planteadas.

Los artículos seleccionados fueron revisados y se les aplica una plantilla que permita uniformizar la calidad y realizar los análisis posteriores ${ }^{23,} 24$ (Apéndice).Los criterios de calidad exigidos para incluirlos en el análisis fueron:

a. Ensayos aleatorios doble ciego

b. Con selección de casos entre una población con signos de envejecimiento. 
c. Con una medida de resultado estandarizada.

d. Con un seguimiento de los pacientes e identificación de las perdidas.

e. Con identificación de las complicaciones.

f. Idioma original Ingles o español.

Los estudios fueron evaluados por los autores del trabajo. Para garantizar la uniformidad en la evaluación de la calidad de los artículos se establece un cuestionario en forma de "ficha" que constituye la base de la recolección de la información que se utilizara en el metaánalisis y constituye el del nominado análisis cuantitativo. La información obtenida, una vez cumplimentadas las fichas, permite decidir que artículos pasan a la segunda fase en función de su calidad. La calidad se toma por acuerdo de los investigadores.

\section{Los análisis estadísticos}

Se ha utilizado el método de efectos fijos de Mantel y Haenszel para combinar los resultados de los diferentes estudios ${ }^{25}$. En los casos en los que ha sido posible se realizan análisis de sensibilidad para comprobar la robustez de los datos obtenidos.

\section{Resultados de la búsqueda}

El proceso de selección de los artículos incluidos el estudio se representa en el Apéndice 1.

Se han encontrado 148 artículos potencialmente útiles con los criterios de ser ensayos clínicos en humanos, todos ellos con abstract disponible. A estos 148 artículos se han añadido 4 artículos más de fuentes secundarias (encontrados en las referencias) con lo que el total de artículos analizados ha sido de 152 artículos, todos ellos incluidos en el listado bibliográfico de artículos revisados. Tras la primera evaluación de los abstracts por parte de los investigadores se seleccionaron 25 artículos para su revisión completa y quedaron incluidos en el estudio, se agruparon en función de su objetivo en los distintos apartados de la Tabla 1.

La temática mas frecuentemente analizada han sido los artículos centrados en la distribución de la grasa corporal aunque la mayor parte de los artículos trataban mas de una temática mezclando frecuentemente las determinaciones de niveles hormonales (DHEA, DHEA/S, Testosterona y GFH-I) y estudios sobre la distribución de la grasa corporal y perfiles lipidicos.

Los resúmenes de los artículos revisados y sus características se agrupan en la Tabla 2 .
Apéndice 1. Ficha de evaluación de artículos

Ficha numero .................

\begin{tabular}{ll}
\hline Articulo $\mathbf{N}^{\circ}$ & Comentarios
\end{tabular}

Fecha

Cual es el objetivo

Como mide el efecto del fár-

maco

Son los grupos aleatorios.

si no

Distribuidos al azar

Tienen alguna limitación para ser tratados, es decir no reflejan la población general de su misma edad y genero.

Es un estudio ciego pacientes, el paciente no sabe lo que si no toma

Es un estudio ciego para investigadores si no

Dosis vía duración del tratamiento antes de medir el efecto

Tiempo de seguimiento, mes o años

Perdidas de casos durante el estudio, pacientes que han iniciado el tratamiento pero que no han sido seguidos, si no se comenta hacerlo constar es un mal asunto

Porcentaje de perdidas en total, en tratados en no tratados

Proporción de mujeres

Numero de pacientes participantes. Numero total de pacientes en el estudio

Numero de pacientes que reciben DHEA

Numero de pacientes que no lo reciben o toman placebo

Proporción de mejoría en los tratados

Proporción de mejoría en los no tratados

Valor de efecto en tratados

Valor de efecto en no tratados 
Tabla 1. Artículos analizados y su objetivos de estudio

\section{Objetivos de los estudios}

Aspectos generales

Composición corporal

Masa osea

Grasa corporal y perfiles lipidicos

Tolerancia Hidratos de carbono

Niveles hormonales

Otros efectos

Inmunocompetencia

Actividad Fibrinolitica

Actividad endotelial

Percepción estado

54, 72,94,116,133,149, 151

7

Memoria

$31,67,72,149$

4

$54,61,72,133,147,149$
Número

35,99

\section{2}

4

6

$41,62,69,77,142,146,151$

$30,89,94,99,133,142,147,149 \quad 8$

$120,41,122,133$
Actividad sexual

Tabla 2. Características y objetivos de los artículos analizados

\begin{tabular}{|c|c|c|c|c|c|c|c|}
\hline & $\begin{array}{l}\text { Articulo } \\
\mathbf{N}^{\circ}\end{array}$ & $\begin{array}{l}\text { Selección } \\
\text { Casos }\end{array}$ & Casos & $\begin{array}{l}\text { Tratamiento } \\
\text { Dosis/dia }\end{array}$ & Control & Seguimiento & Evaluación \\
\hline 1 & 6 & Aleatorio & 50 mujeres & $50 \mathrm{mg}$ & Grupo placebo & 12 semanas & $\begin{array}{l}\text { Perímetro pantorrilla } \\
\text { Lípidos } \\
\text { Sensación bienestar }\end{array}$ \\
\hline 2 & 30 & Aleatorio cruzado & 10 mujeres & $50 \mathrm{mg}$ & Fase placebo & 8 días & Niveles Hormonales \\
\hline 3 & 31 & Aleatorio cruzado & 6 mujeres & $50 \mathrm{mg}$ & Fase placebo & 28 días & $\begin{array}{l}\text { Niveles } \\
\text { Hormonales } \\
\text { Nivel Cognitivo }\end{array}$ \\
\hline 4 & 41 & & 24 varones & $25 \mathrm{mg}$ & Placebo & 12 semanas & $\begin{array}{l}\text { Función endotelial } \\
\text { Sensibilidad insulina }\end{array}$ \\
\hline 5 & 54 & Aleatorio cruzado & 16 mujeres & $300 \mathrm{mg}$ & Fase placebo & $60 \mathrm{~min}$ & Libido \\
\hline 6 & 55 & Aleatorio cruzado & 43 varones & $90 \mathrm{mg}$ & Fase placebo & 6 meses & Metabolismo óseo \\
\hline 7 & 61 & Aleatoria & 85 varones & $50 \mathrm{mg}$ & Grupo placebo & 6 meses & Disfunción eréctil \\
\hline 8 & 62 & Aleatorio & 20 mujeres & $25 \mathrm{mg}$ & Grupo placebo & 12 meses & $\begin{array}{l}\text { Sensibilidad } \\
\text { Insulina } \\
\text { Perfil lipidico }\end{array}$ \\
\hline 9 & 67 & Aleatoria & 46 varones & $50 \mathrm{mg}$ & & 5 meses & $\begin{array}{l}\text { Nivel Cognitivo } \\
\text { Bienestar }\end{array}$ \\
\hline 10 & 69 & Aleatorio & 24 mujeres & $50 \mathrm{mg}$ & Grupo placebo & 5 meses & $\begin{array}{l}\text { Leptinemia } \\
\text { Glucemia } \\
\text { Masa ósea }\end{array}$ \\
\hline 11 & 72 & Aleatoria & 24 mujeres & $50 \mathrm{mg}$ & Grupo placebo & 5 meses & $\begin{array}{l}\text { Bienestar } \\
\text { Nivel Cognitivo } \\
\text { Sexualidad }\end{array}$ \\
\hline 12 & 77 & Aleatorio cruzado & 9 mujeres & $\begin{array}{l}50 \mathrm{mg} \\
9 \text { hombres }\end{array}$ & Fase placebo & 6 meses & $\begin{array}{l}\text { Composición } \\
\text { Corporal } \\
\text { Masa ósea } \\
\text { Tolerancia glucosa } \\
\text { Niveles de IGF } \\
\text { Niveles Estrógenos } \\
\text { Perfil Lipidico }\end{array}$ \\
\hline 13 & 89 & Aleatorio & $\begin{array}{l}24 \text { varones } \\
14 \text { mujeres }\end{array}$ & $50 \mathrm{mg}$ & Grupo placebo & & Esteroides urinarios \\
\hline
\end{tabular}


Tabla 2 (Continuación)

\begin{tabular}{|c|c|c|c|c|c|c|c|}
\hline & $\begin{array}{l}\text { Artículo } \\
\mathbf{N}^{\circ}\end{array}$ & $\begin{array}{l}\text { Selección } \\
\text { casos }\end{array}$ & Casos & $\begin{array}{l}\text { Tratamiento } \\
\text { Dosis/dia }\end{array}$ & Control & Seguimiento & Evaluación \\
\hline 14 & 94 & Aleatorio & 60 mujeres & $50 \mathrm{mg}$ & Grupo placebo & 3 meses & $\begin{array}{l}\text { Percepción salud } \\
\text { Cambios hormonales } \\
\text { Perfil Lipidico }\end{array}$ \\
\hline 15 & 99 & Aleatorio cruzado & 14 varones & $50 \mathrm{mg}$ & Fase placebo & $\dot{\delta}$ & Cambios hormonales \\
\hline 16 & 116 & Aleatorio cruzado & $\begin{array}{l}45 \text { varones } \\
15 \text { mujeres }\end{array}$ & $50 \mathrm{mg}$ & Fase placebo & 2 semanas & $\begin{array}{l}\text { Depresión Escala } \\
\text { Calidad de vida } \\
\text { Cuestionario Humor }\end{array}$ \\
\hline 17 & 120 & Aleatorio cruzado & 9 varones & $50 \mathrm{mg}$ & Fase placebo & 22 semanas & Inmunocompetencia \\
\hline 18 & 122 & Aleatorio & 34 varones & $50 \mathrm{mg}$ & Grupo placebo & 12 días & Act. fibrinolitica \\
\hline 19 & 133 & Aleatorio & $\begin{array}{l}13 \text { varones } \\
17 \text { mujeres }\end{array}$ & $50 \mathrm{mg}$ & Con basal & 6 meses & $\begin{array}{l}\text { Cambios hormonales } \\
\text { Perfil Lipidico } \\
\text { Masa corporal } \\
\text { Libido } \\
\text { Bienestar }\end{array}$ \\
\hline 20 & 142 & Aleatorio cruzado & 6 mujeres obesas & $1.600 \mathrm{mg}$ & Fase placebo & 28 días & $\begin{array}{l}\text { Andrógenos } \\
\text { Perfil Lipidico } \\
\text { Tolerancia Glucosa } \\
\text { Grasa corporal }\end{array}$ \\
\hline 21 & 146 & Aleatorio & $\begin{array}{l}28 \text { varones } \\
28 \text { mujeres }\end{array}$ & $50 \mathrm{mg}$ & Grupo placebo & 6 meses & $\begin{array}{l}\text { Grasa Abdominal } \\
\text { Tolerancia Glucosa }\end{array}$ \\
\hline 22 & 147 & Aleatorio cruzado & 39 varones & $100 \mathrm{mg}$ & Fase placebo & 3 meses & $\begin{array}{l}\text { Grasa Corporal } \\
\text { Actividad sexual } \\
\text { Cambios hormonales }\end{array}$ \\
\hline 24 & 151 & Aleatorio & $\begin{array}{l}30 \text { varones } \\
30 \text { mujeres } \\
30 \text { varones control } \\
30 \text { mujeres control }\end{array}$ & $\begin{array}{l}75 \mathrm{mg} \text { varones } \\
50 \mathrm{mg} \text { mujeres }\end{array}$ & Grupo placebo & 24 meses & $\begin{array}{l}\text { Masa Coporal } \\
\text { Densitometria } \\
\text { Perfil Lipidico } \\
\text { Loterancia Insulina } \\
\text { Capacidad física } \\
\text { Percepción salud }\end{array}$ \\
\hline 25 & 152 & Aleatorio & $\begin{array}{l}70 \text { varones } \\
70 \text { mujeres }\end{array}$ & $50 \mathrm{mg}$ & Grupo placebo & 12 meses & Densitometria \\
\hline
\end{tabular}

Se han revisado 25 artículos todos ellos ensayos clínicos doble ciego. En algunos los casos se dividen en dos grupos seleccionados aleatoriamente, los que reciben tratamiento con DHEA y los que reciben placebo. En otros artículos hay un solo grupo de casos con la adscripción aleatoria al grupo de pacientes tratados, o bien a los no tratados durante el tiempo del estudio (estudios cruzados). Esta forma de estudio es la más frecuente en los artículos analizados.

De los 855 pacientes reclutados 315 eran mujeres y 510 varones todos con niveles bajos de DHEA.

Las dosis suministrada en la mayoría de los trabajos estudiados era de $50 \mathrm{mg}$ orales por día, únicamente dos estudios utilizaban dosis inferiores $(25 \mathrm{mg}$ ) y cinco estudios dosis superiores, con la dosis superior encontrada de $1600 \mathrm{mg}$. Los periodos de seguimientos antes de evaluar los efectos en la mayoría de los casos era como mínimo de cuatro semanas, y solamente algunos estudios interesados en niveles hormonales o efectos sobre la libido hacían las evaluaciones a más corto plazo

\section{RESULTADOS SOBRE CAMBIOS OBJETIVOS DE LA REVISIÓN \\ Efectos de la DHEA sobre los niveles hormonales de andrógenos y IGF-I}

El objeto de administrar DHEA/S en pacientes adultos se basa en la reducción fisiológica en la pro- 
ducción de esta hormona en la edad media de la vida y por lo tanto se persigue alcanzar dosis similares a las que se encuentran en adultos jóvenes. Callies F (89) confirma que la dosis de 50mg orales tanto en mujeres como en hombres constituye una dosis suficiente para los objetivos planteados. Dosis superiores, como es la 100mg día, alcanzan niveles suprafisiológicos. La misma información se obtiene de Arlt W (99) que confirma lo dosis de $50 \mathrm{mg}$ como la adecuada para conseguir niveles fisiológicos de DHEA:

Los efectos sobre otras hormonas con las dosis de 50 mg diarios, como los andrógenos, se resumen en la Tabla 3.

Los valores plasmáticos de la DHEAS no han sido expresados en la tabla pues siguen valores paralelos a los de la DHEA. En todos los casos hay un incremento de la DHEA/S tras la administración oral de $50 \mathrm{mg}$ diarios.

La elevación de la testosterona se produce tanto en hombres como en mujeres, aunque el valor estadístico de la elevación es más consistente en las mujeres en los artículos analizados.

La elevación de la IGF-I y la consiguiente reducción de la proteína transportadora se confirman en la mayoría de los artículos analizados y se observa tanto en hombres como en mujeres.

La publicación de Mortola JF, Yen SS (142) no se incluye en el estudio pues la dosis de tratamiento es $1600 \mathrm{mg}$, muy superior a la recomendada para los efectos de antienvejecimiento objeto del estudio en esta revisión.

Tabla 3. Cambios hormonales con la administración de DHEA

\begin{tabular}{|c|c|c|c|c|c|}
\hline Autor (articulo) & Dosis & $\begin{array}{l}\text { DHEA } \\
\mathrm{ng} / \mathrm{mL} \pm \mathrm{ds} \varepsilon\end{array}$ & $\begin{array}{l}\text { Testosterona } \\
\text { (libre) pg/ml } \\
\pm \text { ds } \varepsilon\end{array}$ & Cortisol & $\begin{array}{l}\text { IGF-I } \\
\mathbf{n g} / \mathbf{m l} \\
\pm \mathbf{d s} \varepsilon\end{array}$ \\
\hline $\begin{array}{l}\text { Barnhart KT(94) } \\
\text { Mujeres } 60\end{array}$ & $50 \mathrm{mg}$ & $\begin{array}{l}+242 \% \\
*(i c 9560-423)\end{array}$ & $\begin{array}{l}+94,8 \% \\
*(\text { ic95 34,2, }-155,4)\end{array}$ & $\begin{array}{l}-13,2 \% \\
(\text { ic95 +0,5 -27,8) }\end{array}$ & \\
\hline $\begin{array}{l}\text { Morales AJ(133) } \\
\text { Varones } \\
\text { Mujeres }\end{array}$ & $\begin{array}{l}50 \mathrm{mg} \\
\text { Placebo } \\
50 \mathrm{mg} \\
\text { Placebo }\end{array}$ & $\begin{array}{l}14,72 \pm 1,4 \\
8,47 \pm 0,8 \\
16,13 \pm 1,3 \\
7,17 \pm 0,5\end{array}$ & $\begin{array}{l}20,86 \pm 7,04 \\
15,0 \pm 5,7\end{array}$ & $\begin{array}{l}273 \pm 28,8 \\
245 \pm 36,3\end{array}$ & $\begin{array}{l}181 \pm 15,4^{*} \\
151 \pm 10,2 \\
157,4 \pm 16^{*} \\
140,8 \pm 14\end{array}$ \\
\hline $\begin{array}{l}\text { FlynnMA(146) } \\
\text { Varones } \\
\text { Mujeres }\end{array}$ & $\begin{array}{l}50 \mathrm{mg} \\
\text { Placebo } \\
50 \mathrm{mg} \\
\text { Placebo }\end{array}$ & $\begin{array}{l}5,02 \pm 2,15 \\
2,14 \pm 2,2\end{array}$ & $\begin{array}{l}5,2 \pm 1,1 \\
4,8 \pm 1,4 \\
1,4 \pm 0,5^{*} \\
0,4 \pm 0,4\end{array}$ & & $\begin{array}{l}186 \pm 36^{*} \\
166 \pm 43 \\
188 \pm 61^{*} \\
157 \pm 55\end{array}$ \\
\hline $\begin{array}{l}\text { Wierbeke A,(149) nmol/1 } \\
\text { Varones 50-59 } \\
\text { Varones } 60-69\end{array}$ & $\begin{array}{l}50 \mathrm{mg} \\
\text { Placebo } \\
50 \mathrm{mg} \\
\text { Placebo }\end{array}$ & $\begin{array}{l}19,5 \pm 3,7 \\
10,4 \pm 3,2\end{array}$ & $\begin{array}{l}18,8 \pm 4,7 \\
18,6 \pm 5,2 \\
17,4 \pm 2,4 \\
19 \pm 3,3\end{array}$ & & $\begin{array}{l}18,6 \pm 4 \\
18,5 \pm 4,7\end{array}$ \\
\hline Villareal DT (77) & $\begin{array}{l}50 \mathrm{mg} \\
\text { Placebo }\end{array}$ & & $\begin{array}{l}15,6 \pm 1,8 \\
10,7 \pm 1,2\end{array}$ & & $\begin{array}{l}143 \pm 7^{*} \\
108 \pm 8\end{array}$ \\
\hline $\begin{array}{l}\text { Oliver T (116) } \\
\text { Varones } \\
\text { Mujeres }\end{array}$ & $\begin{array}{l}50 \mathrm{mg} \\
\text { Placebo } \\
50 \mathrm{mg} \\
\text { Placebo }\end{array}$ & $\begin{array}{l}5,86 \pm 0,3 \\
2,85 \pm 0,2 \\
8,1 \pm 0,5 \\
3,5 \pm 0,4\end{array}$ & $\begin{array}{l}6 \pm 0,5^{*} \\
4,575 \pm 0,4 \\
1,2 \pm 0,1^{*} \\
0,19 \pm 0,3\end{array}$ & & $\begin{array}{l}122,3 \pm 8,2 \\
120,3 \pm 6,9 \\
104,1 \pm 6,5 \\
96,3 \pm 9,3\end{array}$ \\
\hline $\begin{array}{l}\text { Narin (151) } \\
\text { Varones }\end{array}$ & $\begin{array}{l}75 \mathrm{mg} \\
\text { Placebo } \\
50 \mathrm{mg}\end{array}$ & $\begin{array}{l}6 \pm 1 \\
7 \pm 1 \\
4,0 \pm 0,5\end{array}$ & & & \\
\hline Mujeres & Placebo & $3,0 \pm 0,5$ & & & \\
\hline
\end{tabular}

* Diferencia con valor estadísticamente significativo, $\mathrm{p}<0,05$.

$\varepsilon$ ds Desviación estándar. 
Efectos de la administración de la DHEA sobre la composición corporal (masa ósea, masa magra, grasa corporal y perfil lipidico) Masa ósea

Los estudios utilizan distintos procedimientos para evaluar la situación del tejido óseo como la densitometría, niveles de calcitonina o metabolitos por lo que resulta imposible acumular los resultados.

La masa ósea medida por densitometría en el trabajo de Villareal DT (77) confirma que tras el tratamiento se mejora tanto la masa ósea total como la masa ósea lumbar, en un 1,6 y un 2,5\% respectivamente aunque la significación clínica de este moderado porcentaje es desconocida. Con densitometría ósea el trabajo de Nair (151) no logra demostrar mejoría en ninguna de las localizaciones estudiadas, columna , muñecas y caderas. En el estudio de Jankowski se demostró mediante densitometría mejoría únicamente en la densidad de la columna en mujeres y en la cadera en mujeres y hombres. Un resultado muy similar en un grupo de mujeres aporta Callies F (69), demostrando un incremento de los niveles de osteocalcina en los pacientes tratados de $1,6 \mathrm{ng} / \mathrm{ml}$ que no fue observado en los controles.

Por otra parte Wiebke Arlt (149) , no fue capaz de demostrar diferencias en los niveles de osteocalcina en los grupos de varones tratado y no tratados, o de los derivados del metabolismo óseo piridinolina o desoxipiridinolina.

Kahn AJ (54), midiendo la procalcitonina, la fosfatasa alcalina especifica del hueso y el péptido del procolageno o la eliminación de hidroxiprolina, no encontró diferencias en dos grupos de pacientes varones tratados con $90 \mathrm{mg}$ al día de DHEA, a pesar de que los niveles de DHEA aumentaron hasta tres veces su nivel basal.

Los estudios revisados resultan contradictorios y en el caso de existir modificaciones en los indicadores del metabolismo óseo, los cambios publicados no parecen tener importancia clínica.

\section{Composición Corporal}

La utilización más difundida del uso de la DHEA/S es, en la actualidad, el incremento de la masa muscular, estando ampliamente difundido su uso en personas de mediana edad. Su uso con el fin de contrarrestar los efectos del envejecimiento paliando los cambios en la composición corporal es el objeto de esta sección. Se han encontrado y analizado 9 artículos que tratan este tema (Tabla 4).

Villareal DT (77) confirma que en pacientes tratados con DHEA se producen incrementos de la masa magra y disminución de la masa grasa según un estudio hecho en dos grupos de pacientes con DHEA baja y tras seis meses de tratamiento.

Pero estos resultados no están confirmados por el estudio de Morales AJ (133). Este no fue capaz de demostrar cambios en la masa corporal grasa en su grupo de pacientes de 40 a 70 años tras seis meses de tratamiento.

Wiebke Arlt (149), en varones con niveles bajos de DHEA, no demostró cambios en la masa corporal. Callies F/69), en 24 mujeres menopáusicas, no consigue demostrar cambios en la distribución corporal. Dayal M (6) no consigue demostrar efecto beneficioso en la masa corporal tras 3 meses de tratamiento en

Tabla 4. Modificaciones de masa magra y grasa

\begin{tabular}{lccccc}
\hline Autor (articulo) & Genero & Masa Magra & Masa Grasa & Casos & Tratamiento \\
\hline Villareal DT (77) & $50 \%$ & Incremento & Disminuye & 18 & $50 \mathrm{mg}$ \\
Morales AJ (133) & $47 \%$ & No cambia & No cambia & 30 & $50 \mathrm{mg}$ \\
Wiebke Arlt (149) & Varones & No cambia & No cambia & 21 & $50 \mathrm{mg}$ \\
Callies F, (69) & Mujeres & No cambia & No cambia & 24 & $50 \mathrm{mg}$ \\
Dayal M, (6) & Mujeres & No cambia & No cambia & 50 & $50 \mathrm{mg}$ \\
Mortola JF, (142) & Mujeres & No cambia & No cambia & 6 & $50 \mathrm{mg}$ \\
M.A. Flynn (147) & Varones & - & No cambia & 39 & $50 \mathrm{mg}$ \\
Dennis T (146) & $50 \%$ & Disminuye & 56 & $50 \mathrm{mg}$ \\
Nair (151) & $50 \%$ & No cambia & No cambia & 60 & $50 / 75 \mathrm{mg}$ \\
\hline
\end{tabular}


50 mujeres. El mismo resultado se obtiene con mujeres obesas en las que el tratamiento no reduce la grasa corporal ni aumenta la masa magra.

Dennis T (146), utilizando la resonancia magnética en un grupo de pacientes tratados (hombres y mujeres),demuestra que existe una reducción de la grasa corporal, tanto subcutánea como visceral.

\section{Perfil Lipidico}

En los pacientes tratados con DHEA/S cabría esperarse una reducción de los niveles de colesterol y HDL, pues bien, los resultados encontrados en los artículos analizados son contradictorios pese a lo uniforme de la técnica de medición (Tabla 5).

M.A. Flynn y Wiebke no observan cambios en la composición corporal de los varones ni en los niveles de lípidos, ni en las HDL ni en el colesterol. Su grupo esta constituido únicamente por hombres. En este mismo subgrupo Morales tampoco encuentra cambios en los perfiles, pero sí en el subgrupo de mujeres en las que evidencia una reducción de los niveles de HDL con el tratamiento. Los dos siguientes estudios realizados en mujeres son contradictorios pues mientras que en el grupo de Lasco existe un incremento de las HDL Mortola y Barnhart KT publican una reducción de hasta el 20\%, si bien este primer grupo esta compuesto de mujeres con sobrepeso, y el segundo por mujeres menopáusicas.
Conclusión: En hombres está claro que el perfil lipídico no cambia y en mujeres el resultado es contradictorio.

\section{Tolerancia a los Hidratos de Carbono}

Los resultados de los estudios centrados en la tolerancia a los hidratos de carbono, a pesar de utilizar metodologías distintas en la evaluación, si muestran una tendencia de resultados mas uniformes. Este tema ha sido analizado en la mayor parte de los estudios de forma colateral y suele estar presente en la mayoría de los trabajos realizados con DHEA. Dentro de los artículos seleccionados ha sido estudiado en 7 de ellos.

Mortola JF (142) encuentra similares niveles de Glucemia e Insulina en ambos grupos pero identifica una mayor respuesta a la Insulina (1126-165 versus 746-165*).

Callies F, No encuentra cambios en la tolerancia a la glucosa, en los niveles de insulina o en la relación Glucosa/Insulina, datos confirmados en el estudio de Villareal DT. y por el estudio de Lasco A. Los pacientes tratados sí muestran mejoría en la sensibilidad a la insulina 1 IndiceM index $+29,55 \%$, $\mathrm{P}=0,01$, sin cambios en la tolerancia a la glucosa.

Dennis $\mathrm{T}$ confirma los mismos resultados en su trabajo aportando la información del área bajo la curva de glucemia e insulina tras sobrecarga, obser-

Tabla 5. Perfiles lipídicos

\begin{tabular}{|c|c|c|c|c|c|c|c|}
\hline \multirow[t]{2}{*}{ Autor (artículo) } & & \multicolumn{2}{|c|}{$\begin{array}{l}\text { Apolipoproteina } \\
\text { Almg/dl } \pm \text { ds } \varepsilon\end{array}$} & \multicolumn{2}{|c|}{$\begin{array}{l}\text { Colesterol } \\
\mathrm{mg} / \mathrm{dL} \pm \mathrm{ds} \varepsilon\end{array}$} & \multicolumn{2}{|c|}{$\begin{array}{l}\text { HDL } \\
\mathrm{mg} / \mathrm{dl} \pm \mathrm{ds} \varepsilon\end{array}$} \\
\hline & & DHEA & Placebo & DHEA & Placebo & DHEA & Placebo \\
\hline M.A. Flynn (147) & Hombres & & & $207 \pm 29$ & $209 \pm 35$ & $50 \pm 11$ & $51 \pm 12$ \\
\hline Wiebke (149) & Hombres & $10,3 \pm 10,3$ & $12,6 \pm 13,3$ & No dif. & No dif. $\phi$ & $57 \pm 18$ & $51 \pm 15$ \\
\hline Morales AJ (133) & $\begin{array}{l}\text { Hombres } \\
\text { Mujeres }\end{array}$ & & & $\begin{array}{l}209 \pm 9,4 \\
218,3 \pm 6,9\end{array}$ & $\begin{array}{l}204 \pm 7 \\
223,7 \pm 5,8\end{array}$ & $\begin{array}{l}44,8 \pm 1,7 \\
63,4 \pm 2,7^{*}\end{array}$ & $\begin{array}{l}45,4 \pm 2,5 \\
68,7 \pm 2,6\end{array}$ \\
\hline Lasco A ( 62) & Mujeres & & & & & $+11,61 * \%$ & \\
\hline Mortola JF (142) & $\begin{array}{l}\text { Mujeres } \\
\text { obesas }\end{array}$ & & & $\begin{array}{l}-11,3 \% \\
\mathrm{p}<0,05\end{array}$ & & $-20,0 \% *$ & \\
\hline Barnhart KT (94) & Mujeres & & & $-2,5 \pm 22,7$ & & $\begin{array}{l}-10,1 \% \pm 1 \\
-5,1)\end{array}$ & \\
\hline Nair (151) & $\begin{array}{l}\text { Mujeres } \\
\text { Hombres }\end{array}$ & & & & & $\begin{array}{l}49 \\
41\end{array}$ & $\begin{array}{l}47 \\
36\end{array}$ \\
\hline
\end{tabular}

* Diferencia con valor estadísticamente significativo, $\mathrm{p}<0,05$.

$\varepsilon$ ds Desviación estándar.

$\phi \mathrm{El}$ estudio no aporta los valores. 
vándose una reducción en el área de la curva de la glucemia y una reducción aunque no significativa en la de insulina, resultados que apoyan la tesis de una mayor sensibilidad a la insulina.

En el estudio de Nair no se demostraron diferencias ni en hombres ni en mujeres en los grupos tratados y controles con respecto a: Glucosa e insulina basales y la sensibilidad a la insulina.

Conclusión: En el metabolismo de los hidratos de carbono sí parece claro que la administración de DHEA produce una mayor sensibilidad a la insulina, aunque en algún estudio no se ha encontrado este hallazgo.

Percepción del estado de salud: La utilización de la DHEA para conseguir una mejoría en la percepción del estado de salud en sus múltiples facetas, como en el humor, síntomas de depresión, grado de actividad física e intelectual, deseo y actividad sexual, ha sido una de las áreas de mayor interés y su uso esta extendido en este ámbito.
La evaluación del efecto sobre la percepción del estado de salud, capacidad física, sexual e intelectual plantea problema de agregación de resultado entre los diferentes estudios por las diferencias en las metodologías utilizadas (Tabla 6).

Barnhart KT no demuestra en mujeres menopáusicas mejoría del humor, disforia, libido, cognición, memoria, o bienestar.

Los resultados de Hackbert L, con dosis elevadas (300 mg) y en tratamiento agudo,demostraron que las mujeres menopáusicas presentaron un mayor deseo sexual desde el punto de vista físico y mental en el grupo de las tratadas en comparación con el grupo placebo, pero cuando se utilizaron sistemas objetivos(pulso vaginal, perfusión vaginal), no se logró demostrar diferencias entre los grupos.

Arlt W(72), con tratamiento de 50 mg, no logró demostrar mejoría en los aspectos cognitivos pero si mejoraron los tests de estado general y de depresión, junto con los marcadores de deseo sexual y satisfacción sexual.

Wolf (116), tanto en varones como en mujeres, no logra demostrar efecto alguno en los tests cognitivos visuales, de memoria, o en la percepción de bienestar. Estos resultados fueron tanto para los hombres como para las mujeres. Morales AJ(133) encuentra una mejoría en la percepción del estado general tanto en hombres como en mujeres con respeto al placebo mientras que no hay cambios en la libido. Los cambios en la mejoría de la percepción del bienestar los relaciona con las elevaciones demostradas en su grupo de la IGF-I y las disminuciones de su proteína transportadora.

No dif. No dif.

No dif. No dif

No dif. No dif

No dif. No dif

No dif. No dif

No dif. No dif.

No dif. No dif.

Depresión No dif. No dif.

$\begin{array}{lllll}\text { Morales AJ(133) } & \text { Varones } & \text { Cognitivo } & & \\ & \text { Mujeres } & \text { Libido } & \text { No dif. } & \text { No dif. } \\ & & \text { Percepción estado } & & \text { Mejoría } \\ & \text { Depresión } & & \text { Mejoría }\end{array}$

Flynn MA (147) con dosis de $100 \mathrm{mg}$ no logra demostrar diferencias en la libido medida con un cuestionario de la actividad sexual.

Wiebke Arlt (149), en varones, no consiguió demostrar efectos beneficiosos del tratamiento con $50 \mathrm{mg}$ de DHEA en los ámbitos de la percepción del estado general utilizando cuestionarios sobre el humor, 
depresión y sintomas. Los efectos sobre la sexualidad fueron medidos con cuatro diferentes cuestionarios sobre frecuencia, deseo y satisfacción sin lograr demostrar diferencias entre tratados y placebo.

Hirshman E, (31), en este trabajo, analiza la relación de los andrógenos y los estrógenos, en mujeres tratadas con $50 \mathrm{mg}$ de DHEA, sobre los niveles cognitivos y se identifica los estrógenos como favorecedores de la cognición mientras que los niveles de andrógenos como ejerciendo efectos negativos sobre la misma lo que explicaría los datos contradictorios de los estudios de pacientes tratados con DHEA.

Van Niekerk JK (67) no logra demostrar beneficio en la percepción del estado de bienestar o de la memoria en varones.

En varones con disfunción eréctil solo parece tener resultados positivos en los que presentan la hipertensión como causa orgánica y también en los que no se identifica causa orgánica. Reiter WJ (61)

En el estudio de Nair(151) utilizando los cuestionarios SF-36 de percepción de estado físico y mental no se demostró mejoría en los grupos de mujeres $\mathrm{u}$ hombres tratados.

Conclusión: Los resultados sobre la percepción física solo ha podido ser demostrado en un grupo de mujeres, las capacidades cognitivas no se modifican, la libido no se modifica con el tratamiento ni en hombres ni en mujeres.

\section{DISCUSIÓN Y CONCLUSIONES}

Las revisiones sistemáticas y la utilización de la técnica de meta-análisis están sometidas a un sin número de sesgos ya desde la fase de búsqueda por importantes condicionantes de la publicación hasta por la pretensión de agrupar estudios muy heterogéneos. En el presente estudio no hemos utilizado ninguna técnica para valorar estos posibles factores, como podría haber sido análisis de sensibilidad o análisis por grupos. Estas limitaciones hacen que las conclusiones deban ser valoradas en función de la calidad de los trabajos.

Las revisiones sistemáticas encontradas ${ }^{26}$ (148) coinciden con las conclusiones extraídas de los datos de la presente revisión.

La DHEA no parece tener efectos sobre los aspectos cognitivos o de la percepción de bienestar ni sobre la actividad sexual, sí parece producir un efecto en el metabolismo de los hidratos de carbono aumentando la eficacia de la insulina, y no se ha conseguido demostrar un efecto sobre el metabolismo lipídico.

\section{REFERENCIAS}

1. Ibanez L, Dimartino-Nardi J, Potau N, Saenger P. Premature adrenarche -norrmal variant or forerunner of adult disease? Endocrine Reviews 2000;21:671-696 .

2. Vermeulen A. Dehydroepiandrosterone sulfate and aging. Annals of the New york Academy of Sciences. 1995;774:121127.

3. Majeswska MD. Neuronal actions of dehydroepiandrosterone. Possible roles in brain development, aging, memory, and effect, Annals of the New York Academy of Sciences. 1995;77:111120.

4. May M, Holmes E, Rogers W, Poth M. Protection from glococorticoid induced thymic involution by dehydroepiandrosterone. Life Science 1991;46:1627-1631.

5. Kimonides VG, Spillantini MG, Sofroniew MV, Fawcett JW, Herbert J. Dehydropiandrosterone (DHEA) antagonises the neurotoxic effects of corticosterone and translocation of SAPK3 in hippocampal primary cultures.Neuroscience 1999;89:429-436.

6. Kimonides VG, Khatabi NH, Svendsen MV, Herbert J. Dehydroepiandrosterone (DHEA) and(DHEA-sulfate(DHEAS) protect hippocampal neurons against excitatory amino acidinduced neurotoxicity. Proceedings of the National Academy of Sciences. USA 1998;95:1852-1857.

7. Lupien SJ, de Leon M, de Santis S, Convit A, Tarshish C, Nair NPV. Cortisol levels during human aging predict hippocampal atrophy and memory deficits. Nature Neuroscience. 1998;1(1): 69-73.

8. Debonnel G, Bergeron R, de Montigny C. Potentiation by dehydroepiandrosterone of the neuronal response to N- methyl-Daspartate in the CA3 region of the rat dorsal hippocampus : an effect mediated via sigma receptors. Journal of Endocrinology. 1996; 150(Supplement):S33-42.

9. Casson PR, Andersen RN, Herrod HG, et al. Oral dehydroepiandrosterone in physiologic doses modulates immune function in postmenopausal women.Am $\mathrm{J}$ obsted Gynecol. 1993;169:1536-1539.

10. Herbert J. Stress, the brain, and mental illness. BMJ 1997; 315:530-535.

11. Barret- Connor E, Khaw KT, Y en SSC. A prospective study of dehydroepiandrosterone sulfate, mortality and cardiovascular disease. N Engl J Med. 1986;315:1519-1524.

12. Ebeling P, Kiovisto VA. Physiological importance of dehydroepiandrosterone. Lancet 1994; 343:1479-81

13. Van Vollenhoven RF, Engleman EG, McGuire JL. An open study of dehydroepiandrosterone in systemic lupus erythematosus. Arthritis Rheum 1994;37:1305-1310.

14. Hillen T,Lun A, Reischies FM, Borchelt M, Steinhagen TE, Schaub RT. DHEA-S plasma levels and incidence of Alzheimer's disease. Biological psychiatry. 2000;47(2):161-163.

15. Nasman B, Olsson T, Backstrom T, Eriksson S, Grankvist K, Viitanen M, Bucht G. Serum dehydroepiandrosterone sulfate in Alzheimer's disease and in multi-infarct dementia. Biological psychiatry 1991;30(7):684-690.

16. Rudman D, Shetty KR, Mattson DE, Plasma dehydroepiandrosterone sulfate in Nursing home men. J Am Geriatr Soc. 1990;38:421-427.

17. Sunderland T, Merril CR, Harrington MG, Lawlor BA, Molchan $\mathrm{SE}$, Martinez R et al. Reduced plasma dehydroepiandrosterone concentrations in Alzheimer's disease. Lancet 1989;2(8662): 570.

18. Berr C, Lafont S, Debuire B, Dartigues J, Baulieu E. Relationships of dehydroepiandrosterone sulfate in the elderly with functional, psychological, and mental status, and short.term mortality: A French community-based study. Proceedings of the National Academy of Sciances USA1996;93: 13410-13415. 
19. Schneider LS, Hinsey M, Lynes S. Plasma dehydroepiandrosterone sulphate in Alzheimr's Disease. Biological Psychiatry. 1992;31:205-208

20. Wiebke Arlt, Joachim Haas, Frank Callies, Martin Reincke, Doris Hu Bler, Michael Oettel et al. Biotransformation of Oral Dehydroepiandrosterone in Elderly Men: Significant Increase in Circulating Estrogens. J Clin Endocrinol Metab 84:21702176.

21. Dickersin K, Scherer R, Lefebvre C. Identifying relevant studies for systemtic reviews. BMJ 1994;309:1286-1291.

22. Slavin B. Best evidence synthesis: an intelligent alternative to meta-analysis? J Clin Epidemiol. 1995;48:9-18.

23. Moher D, Jadad AR, Nichol G, Penman M, Tugwell P, Walsh S. Assesing the quality of randomized controlled trials: an annotated bibliography of scales and checklists. Control Clin Trials. 1995; 16:62-73.

24. Jüni P, Witschi A, Blosch R, Eggar M. The hazards of scoring the quality of clinical trials for meta-analysis. JAMA 1999;282: 1054-1060.

25. Mantel N, Haenszel W. Statistical aspects of the análysis of data form prospective studies of disease $\mathrm{J}$ Natl Cancer Inst 1959;22:719.
26. Wiebke Arlt, Joachim Haas, Frank Callies, Martin Reincke, Doris Hu Bler, Michael Oettel et al. Biotransformation of Oral Dehydroepiandrosterone in Elderly Men: Significant Increase in Circulating Estrogens. J Clin Endocrinol Metab. 84:21702176.

27. Huppert, FA Van Niekerk JK. Dehydroepiandrosterona (DHEA) supplementation for cognitive function. The Cochrane Data bae of Systematic Reviews 2006; Vol 1.

28. GISEG (Italian Study Group on Geriatric Endocrinology); Valenti G, Denti L, Sacco M, Ceresini G, Bossoni S, Giustina A et al. Consensus Document on substitution therapy with DHEA in the elderly. Aging Clin Exp Res. 2006Aug;18(4):277300 .

Correspondencia autor: Dr. JM. Mendivil Dacal Servicio de Urología. Clínica de la Asunción. Ctra. Izaskun, 9 - 20400 Tolosa. Guipúzcoa Tel.: 943697000

E-mail autor: urologia@clinicadelaasuncion.com Información artículo: Revisión - Andrología

Trabajo recibido: septiembre 2008

Trabajo aceptado: octubre 2008 